Document downloaded from:

http://hdl.handle.net/10251/47496

This paper must be cited as:

Querol Vives, A.; Gallardo Bermell, S.; Ródenas Diago, J.; Verdú Martín, GJ. (2011).

Parametric study of the X-ray primary spectra obtained with the MTSVD unfolding method. Applied Radiation and Isotopes. 69(8):1112-1117. doi:10.1016/j.apradiso.2010.10.018.

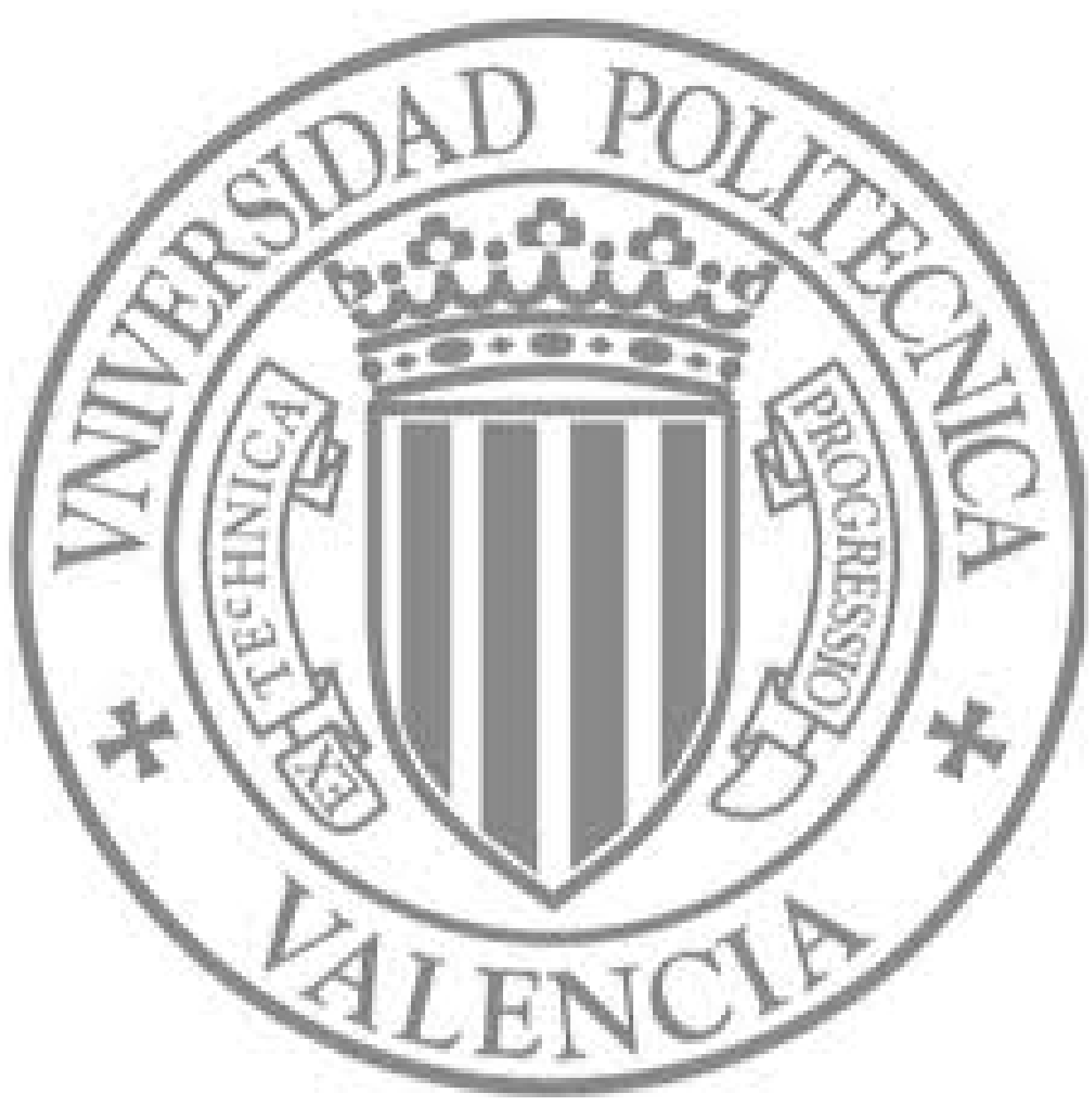

The final publication is available at

http://dx.doi.org/10.1016/j.apradiso.2010.10.018

Copyright Elsevier 


\section{Elsevier Editorial System(tm) for Applied Radiation and Isotopes Manuscript Draft}

Manuscript Number:

Title: Parametric study of the X-ray primary spectra obtained with the MTSVD unfolding method Article Type: Special Issue: 6th CHERNE Workshop

Keywords: Quality Control, X-ray tube, IPEM-78, PHD, Unfolding method, Response matrix, MTSVD. Corresponding Author: Mrs Andrea Querol,

Corresponding Author's Institution: Departamento de Ingeniería Química y Nuclear. Universidad Politécnica de Valencia

First Author: Andrea Querol

Order of Authors: Andrea Querol; Sergio Gallardo, Assistant professor; José Ródenas, Full professor; Gumersindo Verdú, Full professor

Abstract: The Modified Truncated Singular Value Decomposition (MTSVD) unfolding method is applied to obtain primary spectra for X-ray tubes in radiodiagnostic. Three parameters - voltage, anode angle and filter thickness- of the tube are tested. Unfolded spectra are compared with theoretical extracted from IPEM-78 catalogue. A $2 \sigma$ error criterion is applied to assess the minimum variations in tested parameters that permits distinguishing between close spectra. 


\section{UNIVERSIDAD POLITÉCNICA DE VALENCIA}

Departamento de Ingeniería Química y Nuclear.

Editor

Applied Radiation and Isotopes

Special issue for 6th CHERNE workshop

Valencia, 15 July 2010

Dear Sir:

I am pleased to address you the manuscript entitled:

\section{Parametric study of the X-ray primary spectra obtained with the MTSVD unfolding method}

to be published in the special issue of the A Applied Radiation and Isotopes. The manuscript has not been submitted to another journal for possible publication. It has been presented at the $6^{\text {th }}$ CHERNE workshop held in Coimbra (Portugal)

Your sincerely,

Andrea Querol

Andrea Querol

Departamento de Ingeniería Química y Nuclear

Tel.: + 34963879631

Universidad Politécnica de Valencia

46022 Valencia (Spain)

FAX: + 34963877639

Email:anquevi@upvnet.upv.es 


\title{
Parametric study of the X-ray primary spectra obtained with the MTSVD unfolding method
}

\author{
A. Querol, S. Gallardo, J. Ródenas, G. Verdú \\ Universidad Politécnica de Valencia \\ Camí de Vera s/n 46022, Valencia, Spain
}

anquevi@upvnet.upv.es, sergalbe@iqn.upv.es, jrodenas@iqn.upv.es, gverdu@iqn.upv.es

\author{
Corresponding author \\ Andrea Querol \\ Departamento de Ingeniería Química y Nuclear \\ Universidad Politécnica de Valencia \\ Camí de Vera s/n 46022, Valencia, Spain \\ Telf.: +34963879631 \\ Email: anquevi@upvnet.upv.es
}

Total number pages of manuscript: 6

Total number of figures: 11 


\section{ABSTRACT}

The Modified Truncated Singular Value Decomposition (MTSVD) unfolding method is applied to obtain primary spectra for X-ray tubes in radiodiagnostic. Three parameters - voltage, anode angle and filter thickness- of the tube are tested. Unfolded spectra are compared with theoretical extracted from IPEM-78 catalogue. A $2 \sigma$ error criterion is applied to assess the minimum variations in tested parameters that permits distinguishing between close spectra. 


\section{1- INTRODUCTION}

$\mathrm{X}$-ray tubes used in radiodiagnostic range (10 - $150 \mathrm{keV})$ require a complex Quality Control (QC) protocol. However, these QC procedures normally do not include any routine characterization of the primary photon beam. Normally, primary spectrum determination is skipped by measuring other QC parameters easier to be obtained in practice (high voltages, half value layer, homogeneity factor, ripple factor, etc).

The use of direct spectrometry for determining primary X-ray spectrum is practically forbidden as detectors cease to work properly at high count rates. To avoid the pile-up effect in the detector produced by a high fluence rate, a Compton spectrometry technique is proposed. In previous works (Gallardo et al., 2004) (Gallardo et al., 2006) authors described a Monte Carlo (MC) model that uses the MCNP5 code (X5 Monte Carlo Team, 2005) to obtain Pulse Height Distribution (PHD) by simulating the Compton spectrometry process.

The relation between the PHD and primary spectrum is defined by a Response function, expressed as a matrix. This Response matrix is ill conditioned and an unfolding method -such as MTSVD- should be applied to obtain the inverse matrix and hence the primary spectrum.

It is necessary to qualify the accuracy of the unfolding method applied, to know whether the primary beam is properly reproduced.

With this goal in mind, small variations in the working conditions are introduced to obtain different PHDs that after being unfolded are compared with reference spectra (IPEM 78 Report Catalogue).

\section{THE MONTE CARLO MODEL}

The MC model developed includes a point source simulating the X-ray focus, the Compton spectrometer (Matscheko, 1998) and a Germanium detector (Canberra, 2009). A layout of the model can be seen in figure 1 .

The Compton spectrometer consists of a shielding chamber, a scattering chamber containing the scattering material (PMMA) and a spectrometer tube with lead collimators.

The Compton scattering process produces an important decrease on the number of photons entering in the spectrometer tube. Therefore, statistics of the simulation is very poor. To improve statistics the geometry is splitted in two parts, as was presented in Gallardo et al., (2004). The final result, obtained with an F8 tally, is the PHD in the detector.

Electron transport has been activated in the model. However, a $10 \mathrm{keV}$ energy cut-off has been applied to limit the total computer time. 


\section{THE RESPONSE FUNCTION}

The relation between PHD and the primary spectrum can be expressed by equation (1):

$\mathrm{R} \overrightarrow{\mathrm{s}}=\overrightarrow{\mathrm{m}}$

where $\vec{s}=\left(s_{1}, \ldots, s_{N}\right)^{T}$ is the unknown primary spectrum, $\vec{m}=\left(m_{1}, \ldots, m_{M}\right)^{T}$ is the PHD recorded in the detector system, and $\mathbf{R}$ is the Response matrix. The Response matrix, $\mathbf{R}$, can be obtained by calculating with MCNP5 the PHDs produced by different monoenergetic primary spectra.

Once $\mathbf{R}$ is known, the equation (1) theoretically permits to obtain the primary spectrum. But as the determination of this matrix is affected by some errors, an approximation $\overrightarrow{\vec{S}}$ to $\vec{S}$ is chosen in such a way that minimizes the 2-norm of the residual vector, as expressed by equation (2).

$\|\mathrm{R} \overrightarrow{\widetilde{s}}-\overrightarrow{\mathrm{m}}\|_{2}$

Since $\mathbf{R}$ is a real MxN matrix, it admits a Singular Value Decomposition (SVD). But $\mathbf{R}$ is rank deficient, so there are many solutions for the Least Squares problem. An optimal solution can be obtained generating a new Response matrix and removing the parts of the solution corresponding to the smallest singular values (Golub and Van Loan, 1996).

Then the MTSVD method can be used to obtain a new matrix, $\mathbf{R}_{\mathbf{k}}$, where $\mathrm{k}$ is the number of singular values of $\mathbf{R}$ (or rank of $\mathbf{R}$ ) that are considered (Forsythe et al., 1977).

\section{RESULTS AND DISCUSSION}

Three parameters of an X-ray tube have been tested: high voltage, anode angle, and filter thickness. The obtained PHDs for $68,69,70,71$ and $72 \mathrm{kVp}$ are represented in figure 2. It can be seen that small variations in voltage produce small changes in PHDs that are more evident at higher energies. The same effect has been observed for higher voltages ( 80 and $90 \mathrm{kVp}$ ).

PHDs obtained for different anode angles $\left(8^{\circ}, 9^{\circ}, 10^{\circ}, 11^{\circ}\right.$ and $\left.12^{\circ}\right)$ and for different filter thickness $(2.5$, $3.5,4.5$ and $5.5 \mathrm{~mm}$ of aluminium) are represented respectively in figures 3 and 4 . Variations in anode angle have less influence on obtained PHDs, while a change in the filter thickness has more importance mostly at low energies.

Once PHD and Response matrix are obtained, the MTSVD method is applied to unfold the PHD obtaining the primary spectrum.

The quality of the result of the MTSVD method strongly depends on the regularization parameter. This parameter can be selected plotting the L-curve, which consists in representing the 2-norm of the solution vector versus the 2-norm of the residual vector. The L-curve obtained is shown in figure 5 . The optimal value for $\mathrm{k}$ is 115 .

Primary spectra obtained with the unfolding method, that is, the unfolded spectra, have been compared with theoretical spectra extracted from the IPEM-78 catalogue. This comparison is shown in figures 6, 7 
and 8 for variations in voltage, anode angle and filter thickness respectively. An error analysis has been performed by calculating the relative error.

Variations in voltage mainly affect spectra at high energy, hence the unfolded spectra taking into account $2 \sigma$ errors bars must not overlap at high energy range. As it can be seen in figure 9 this is possible for a variation of $2 \mathrm{kVp}$ or higher.

The influence of variations in anode angle is less significant and observed only at low energy. With the same criterion unfolded spectra with $2 \sigma$ error bars are represented in figure 10 , where it can be seen that overlapping is not produced for variations of $3^{\circ}$ or more in anode angle.

Finally, variations in filter thickness mostly affect spectra at low energies. Therefore, the overlapping of spectra must be avoided at this energy range. From the representation in figure 11, it can be stated that a minimum change of $2 \mathrm{~mm}$ in the thickness is required.

\section{CONCLUSIONS}

The MTSVD method is adequate to unfold PHDs to obtain primary spectra for X-ray tubes in radiodiagnostic applications.

Variation in three parameters of the X-ray tube -voltage, anode angle and filter thickness- has been tested.

A comparison of unfolded spectra with theoretical ones extracted from IPEM-78 catalogue has been performed using a $2 \sigma$ criterion.

Maximum variations of $2 \mathrm{kVp}$ for voltage, $3^{\circ}$ for anode angle and $2 \mathrm{~mm} \mathrm{Al}$ for filter thickness are required to avoid an overlapping of spectra in the energy range mostly affected.

\section{ACKNOWLEDGMENTS}

This work has been partially supported by the Valencian Region Government under project Grant GVPRE/2008/136, and the Universidad Politécnica de Valencia under project PAID 06-07-3300. 


\section{REFERENCES}

Canberra website, 2009. www.amptek.com

Forsythe, E., Malcolm, M. A., Moler, C. B., 1977. Computer Methods for Mathematical Computations.

Prentice-Hall Englewood Cliffs, New Jersey.

Gallardo, S., Ginestar, D., Verdú, G., Ródenas, J., Puchades, V., Villaescusa, J. I., 2006. X-ray spectrum unfolding using a regularized truncated SVD method. X-ray spectrometry. Vol. 35, 63-70.

Gallardo, S., Ródenas, J., Verdú, G., 2004. Monte Carlo simulation of the Compton scattering technique applied to characterise diagnostic X-ray spectra. Med. Phys. Vol. 31 (7), 2082-2090.

Golub, G. H., Van Loan, C. F., 1996. Matrix Computations. The Johns Hopkins University Press. Third edition.

IPEM Report 78. Catalogue of Diagnostic X-Ray Spectra \& Other Data. Institute of Physics and Engineering in Medicine.

Matscheko, G., 1998. A Compton spectrometer for measurements of primary photon energy spectra from clinical X-ray units under working conditions. Department of radiation physics Linköping University. S581 85. Linköping, Sweden.

X-5 Monte Carlo Team, 2005. MCNP-A general Monte Carlo N-Particle transport code, version 5, Los Alamos National Laboratory, 2003 (revised 10/03/2005). 


\section{FIGURE CAPTIONS}

Fig. 1. Layout of Compton spectrometer.

Fig. 2. PHD obtained for $68,69,70,71$ and $72 \mathrm{kVp}$.

Fig. 3. PHD obtained for $90 \mathrm{kVp}$ varying the anode angle: $8^{\circ}, 9^{\circ}, 10^{\circ}, 11^{\circ}$ and $12^{\circ}$.

Fig. 4. PHD obtained for $100 \mathrm{kVp}$ varying the filter thickness 2.5, 3.5, 4.5 and $5.5 \mathrm{~mm}$ of $\mathrm{Al}$.

Fig. 5. L-curve MTSVD method.

Fig. 6. Comparison between the IPEM 78 catalogue and the unfolded spectra obtained for 68 and $72 \mathrm{keV}$.

Fig. 7. Comparison between the IPEM 78 catalogue and the unfolded spectra obtained for $90 \mathrm{keV} 8^{\circ}$ and $12^{\circ}$.

Fig. 8. Comparison between the IPEM 78 catalogue and the unfolded spectrum obtained for $100 \mathrm{keV} 2.5$ and $5.5 \mathrm{~mm}$ of $\mathrm{Al}$.

Fig 9. Comparison between unfolded spectra for 70 and $72 \mathrm{kVp}$ with their errors.

Fig 10. Comparison between unfolded spectra for $8^{\circ}$ and $11^{\circ}$ with their errors.

Fig 11. Comparison between unfolded spectra for 2.5 and $4.5 \mathrm{~mm}$ of $\mathrm{Al}$ with their errors. 


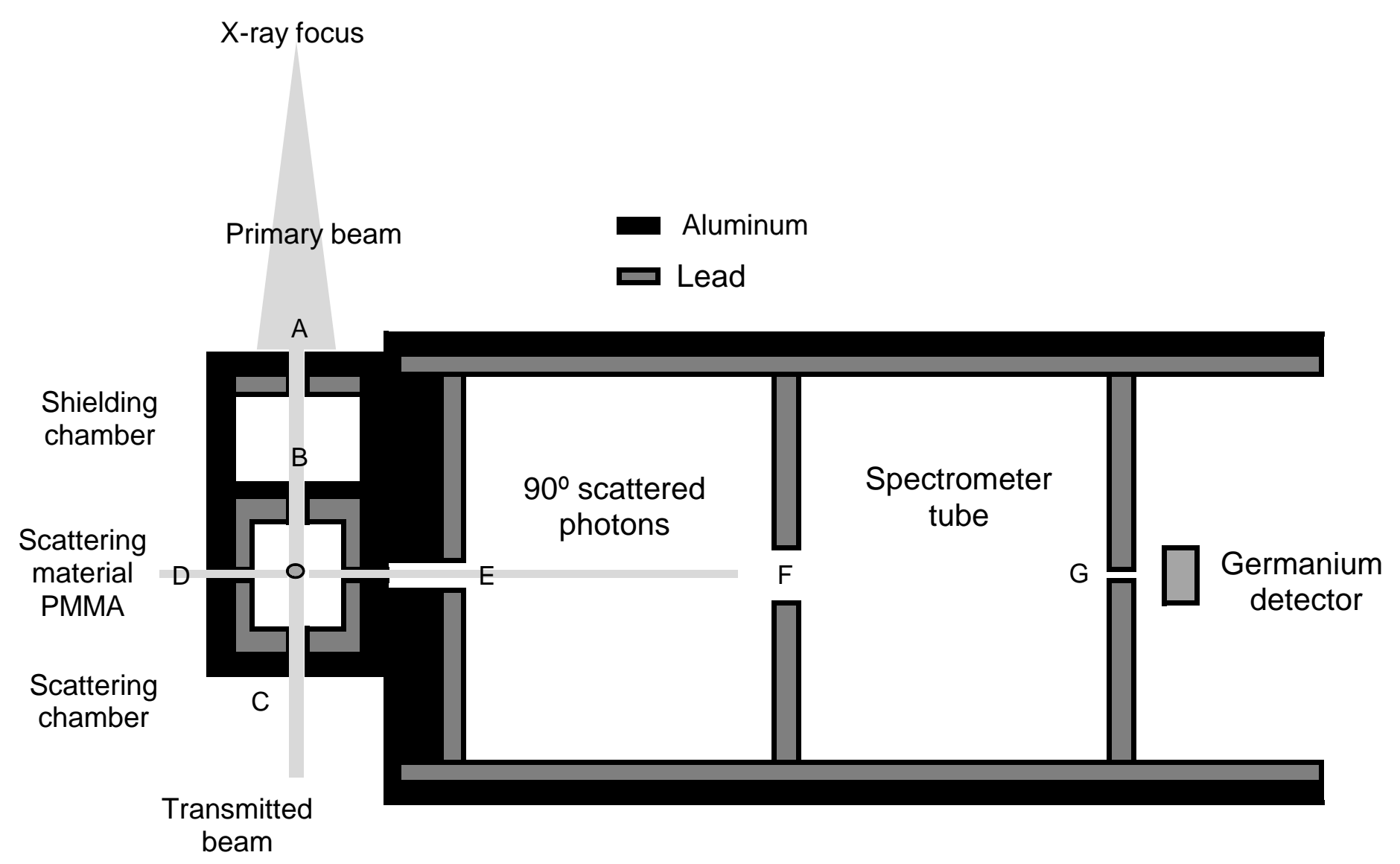




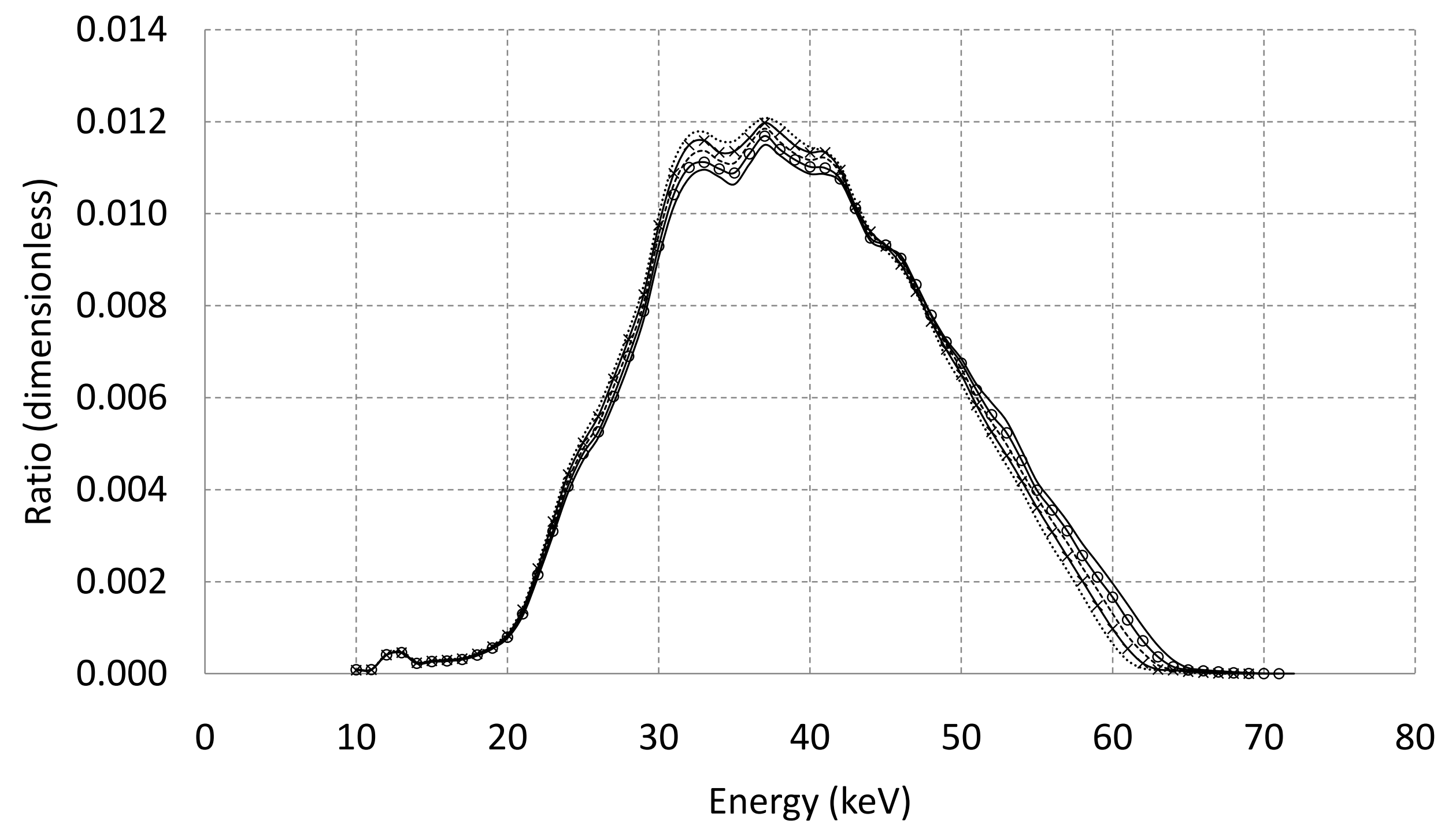

$68 \mathrm{kVp} \rightarrow 69 \mathrm{kVp} \cdots--70 \mathrm{kVp} \rightarrow 71 \mathrm{kVp}-72 \mathrm{kVp}$ 


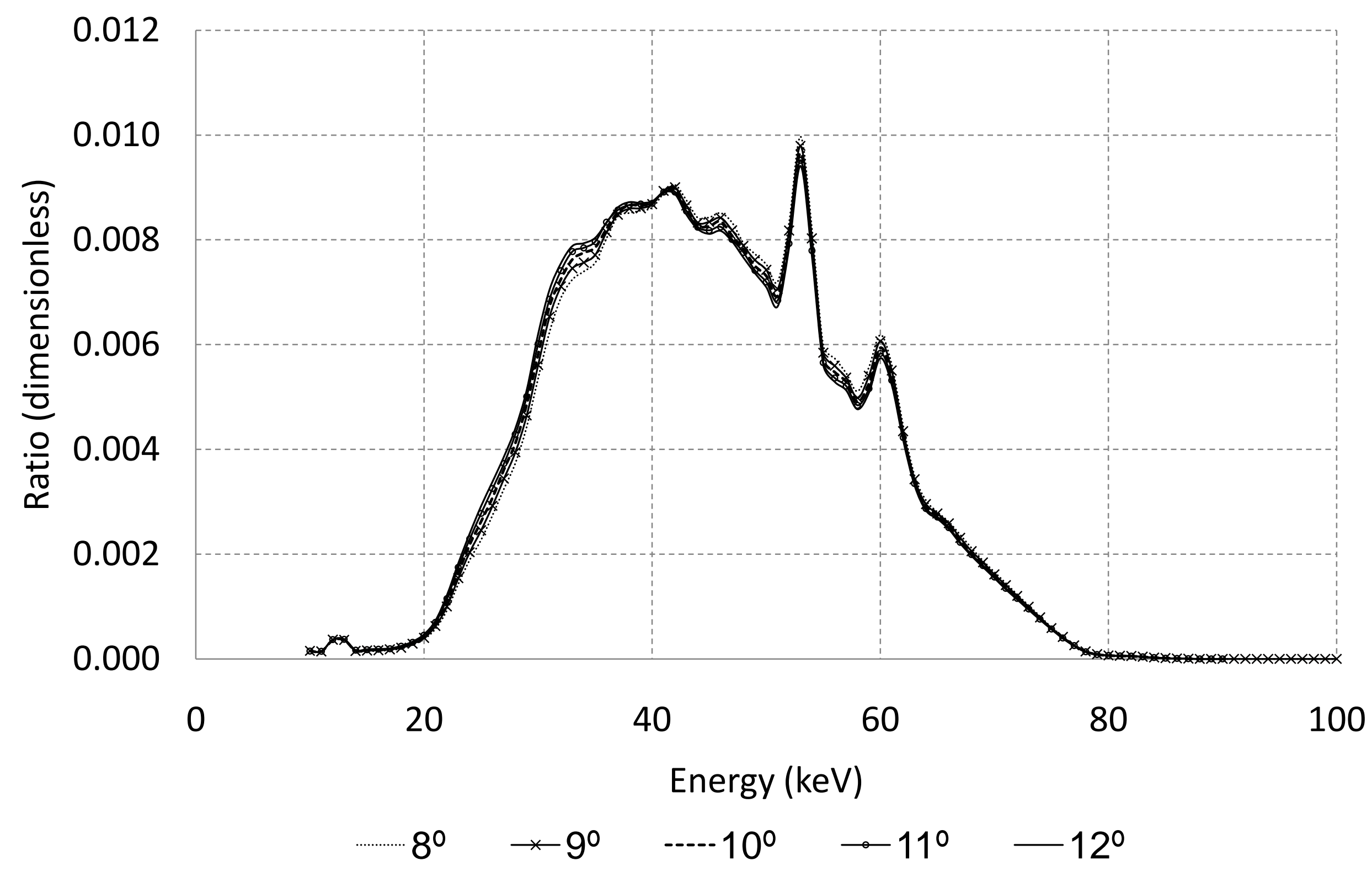




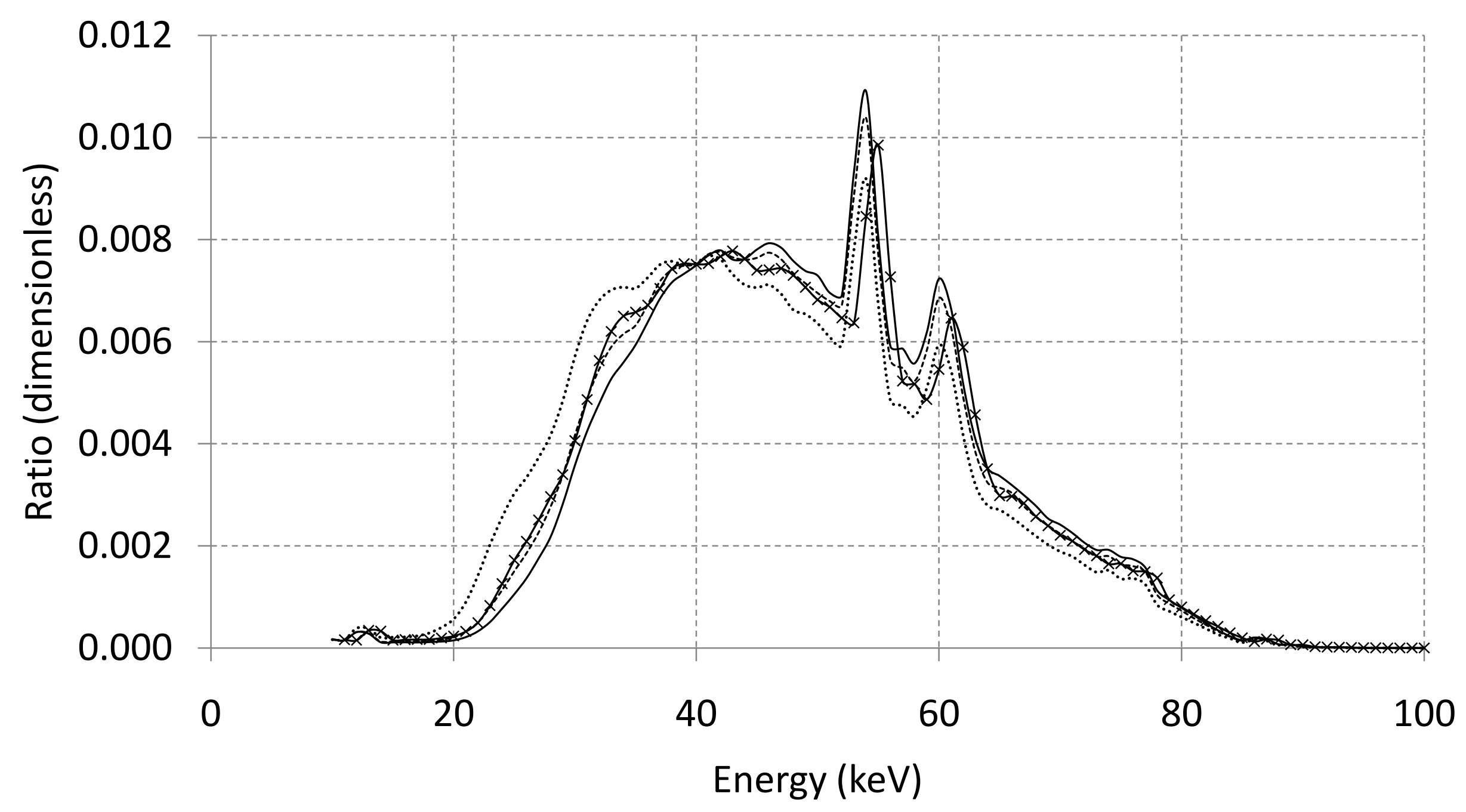

$2,5 \mathrm{~mm} \rightarrow 3,5 \mathrm{~mm} \cdots--4,5 \mathrm{~mm}-5,5 \mathrm{~mm}$ 


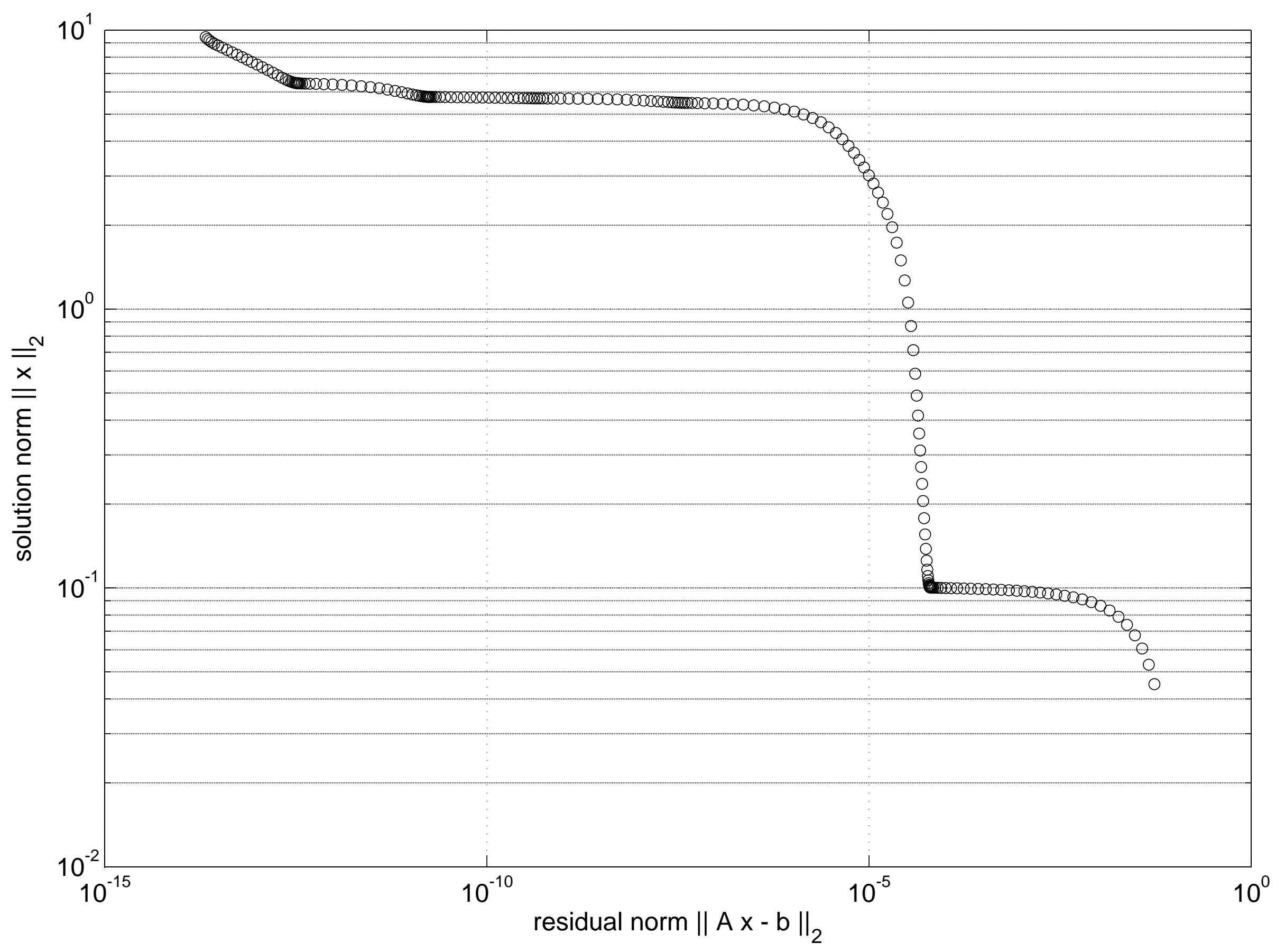




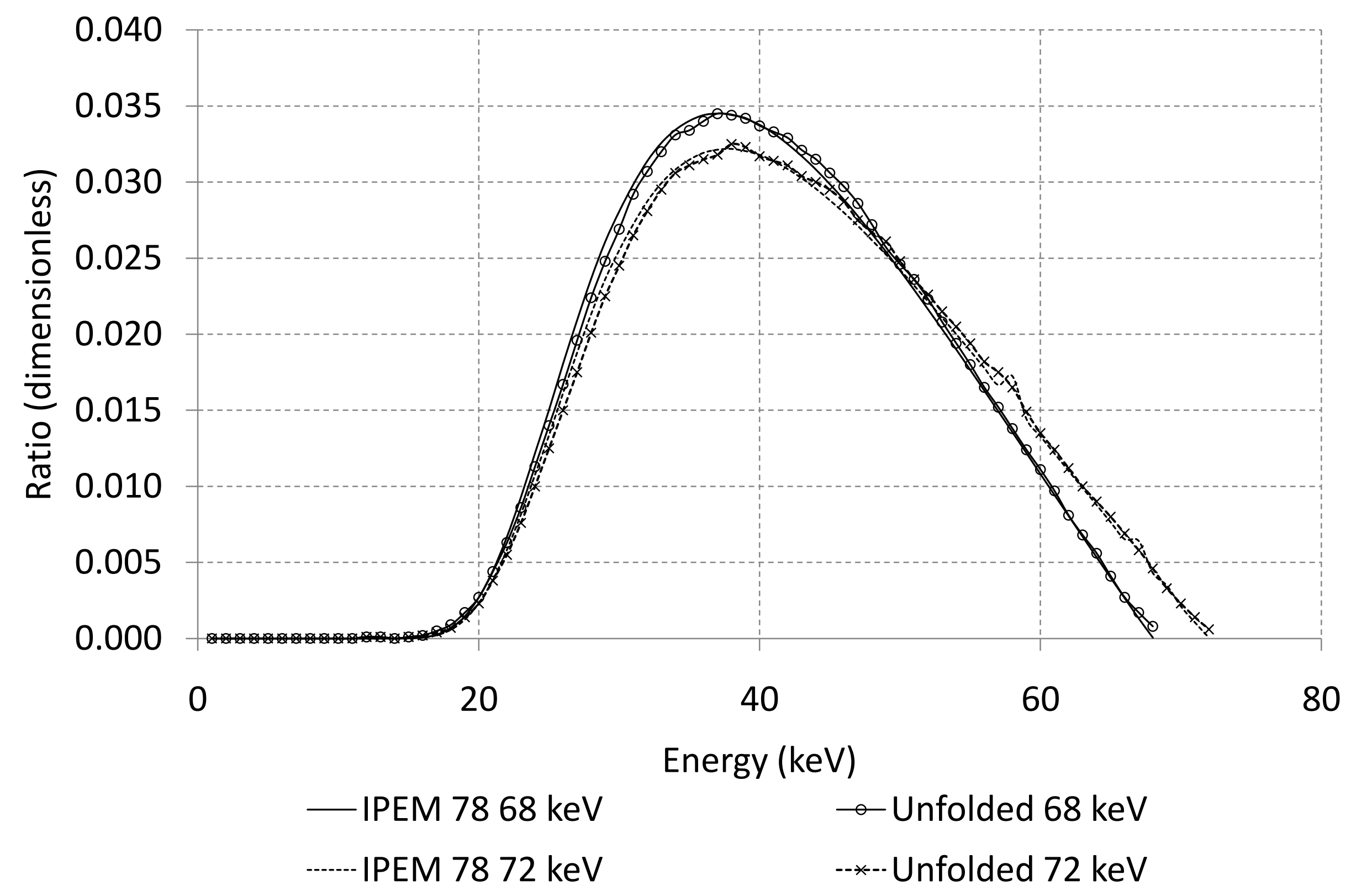




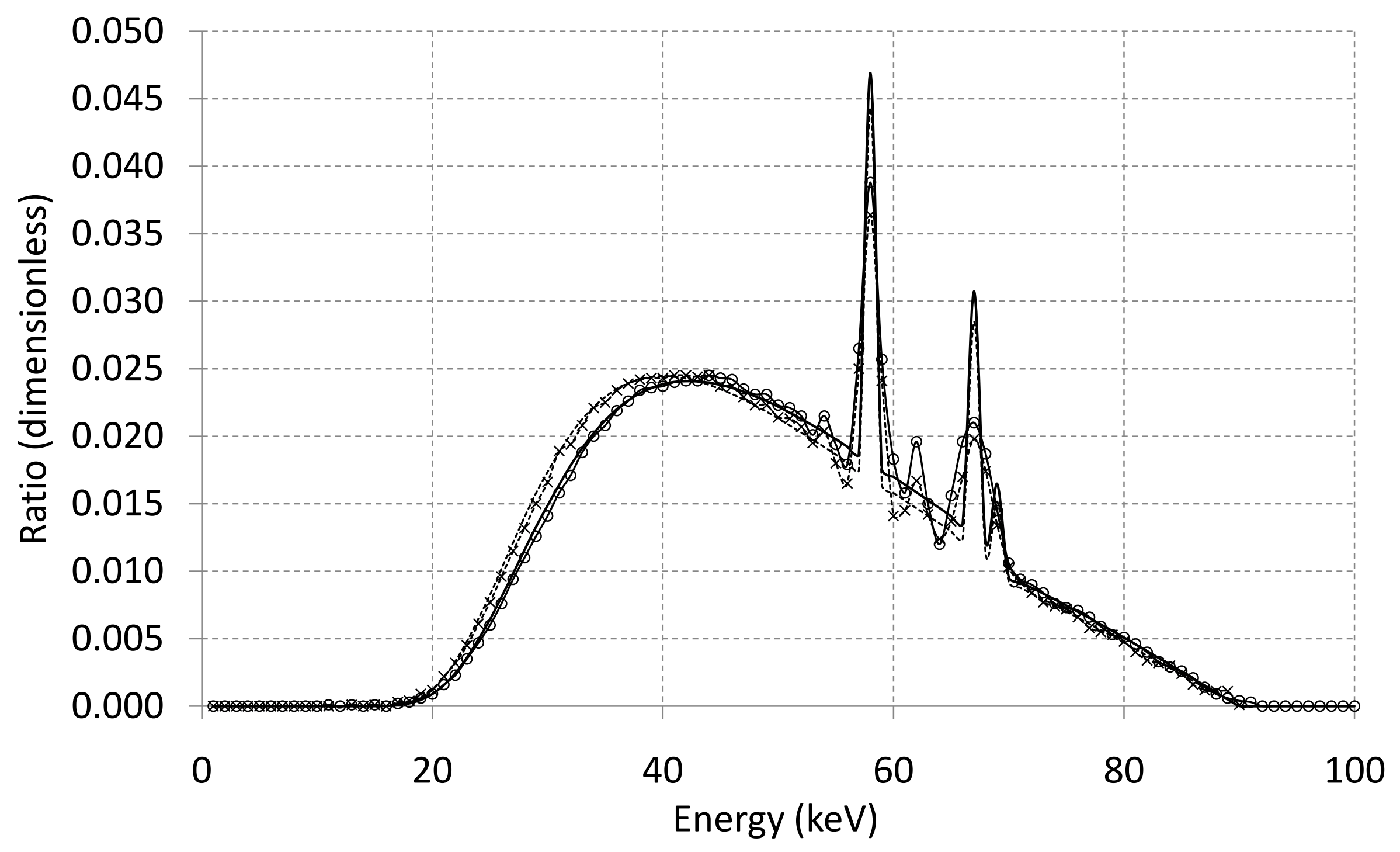

—IPEM 7890 keV 8응 -IPEM $7890 \mathrm{keV} 12^{\circ}$

$\rightarrow$ Unfolded $90 \mathrm{keV} 8^{\circ}$

---x--Unfolded $90 \mathrm{keV} 12^{\circ}$ 


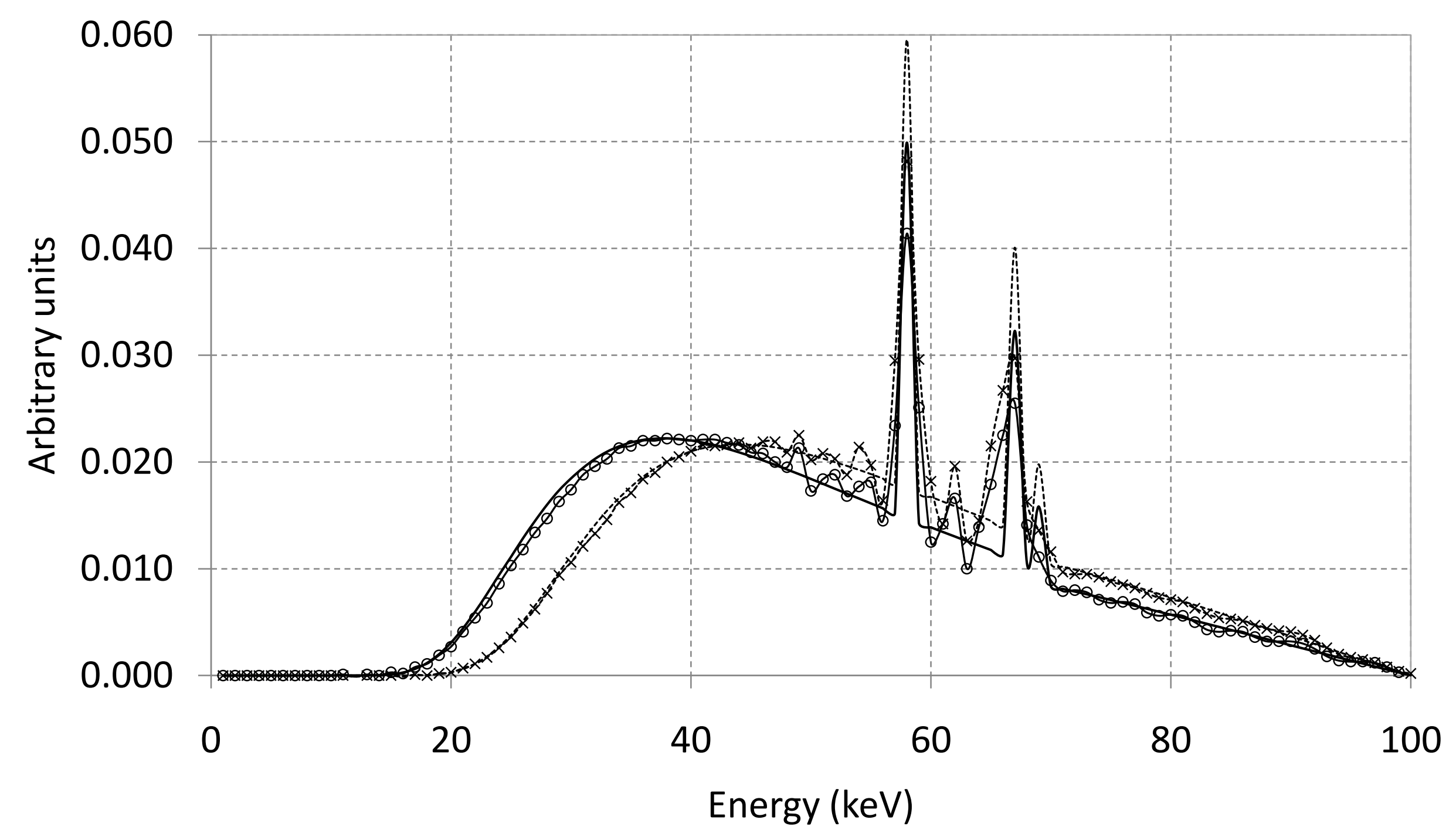

—IPEM $78100 \mathrm{keV} 2.5 \mathrm{~mm} \mathrm{Al}$

$\rightarrow$ Unfolded $100 \mathrm{keV} 2.5 \mathrm{~mm}$ Al -IPEM $78100 \mathrm{keV} 5.5$ mm Al

------ Unfolded $100 \mathrm{keV} 5.5 \mathrm{~mm} \mathrm{Al}$ 


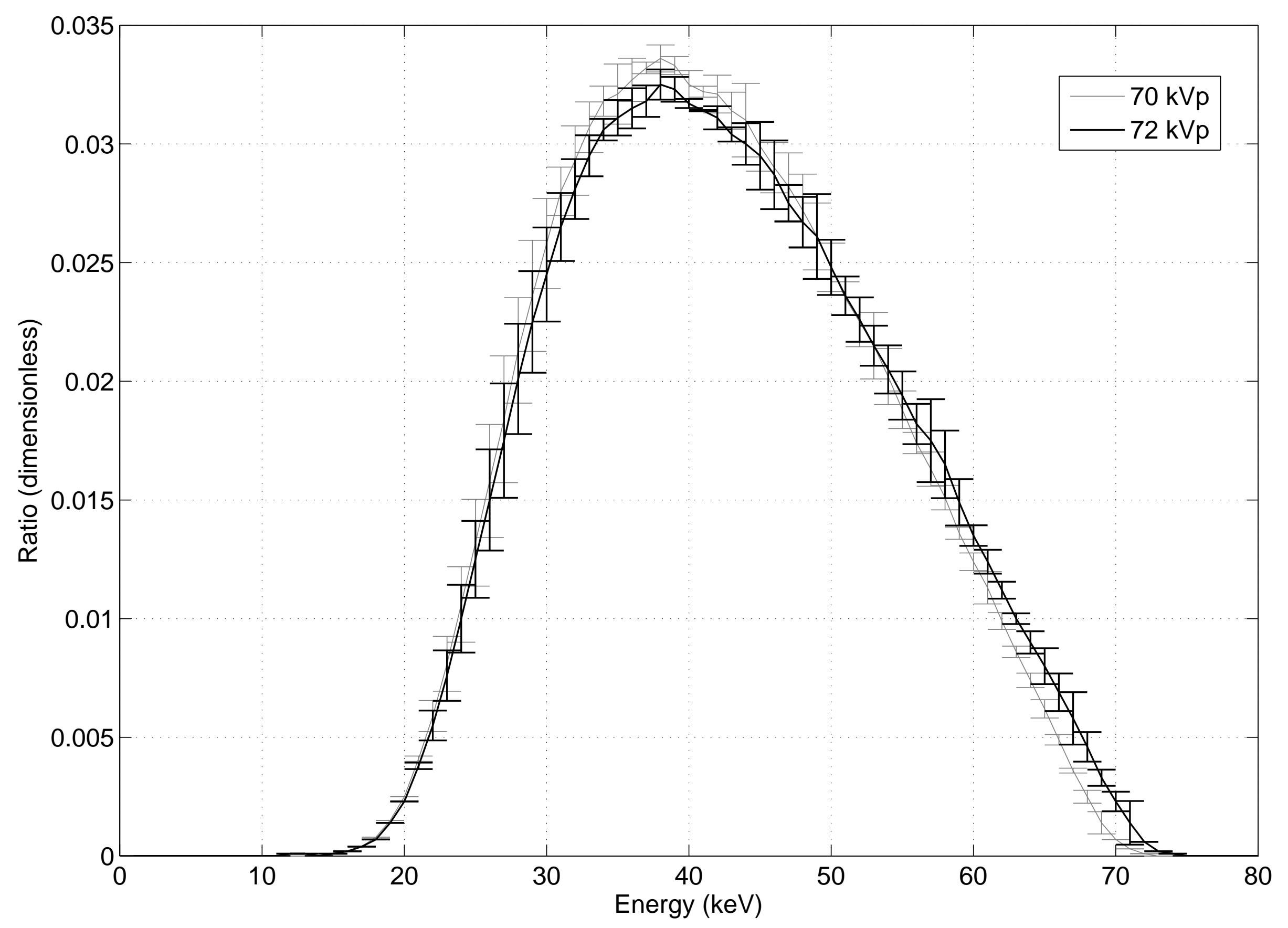




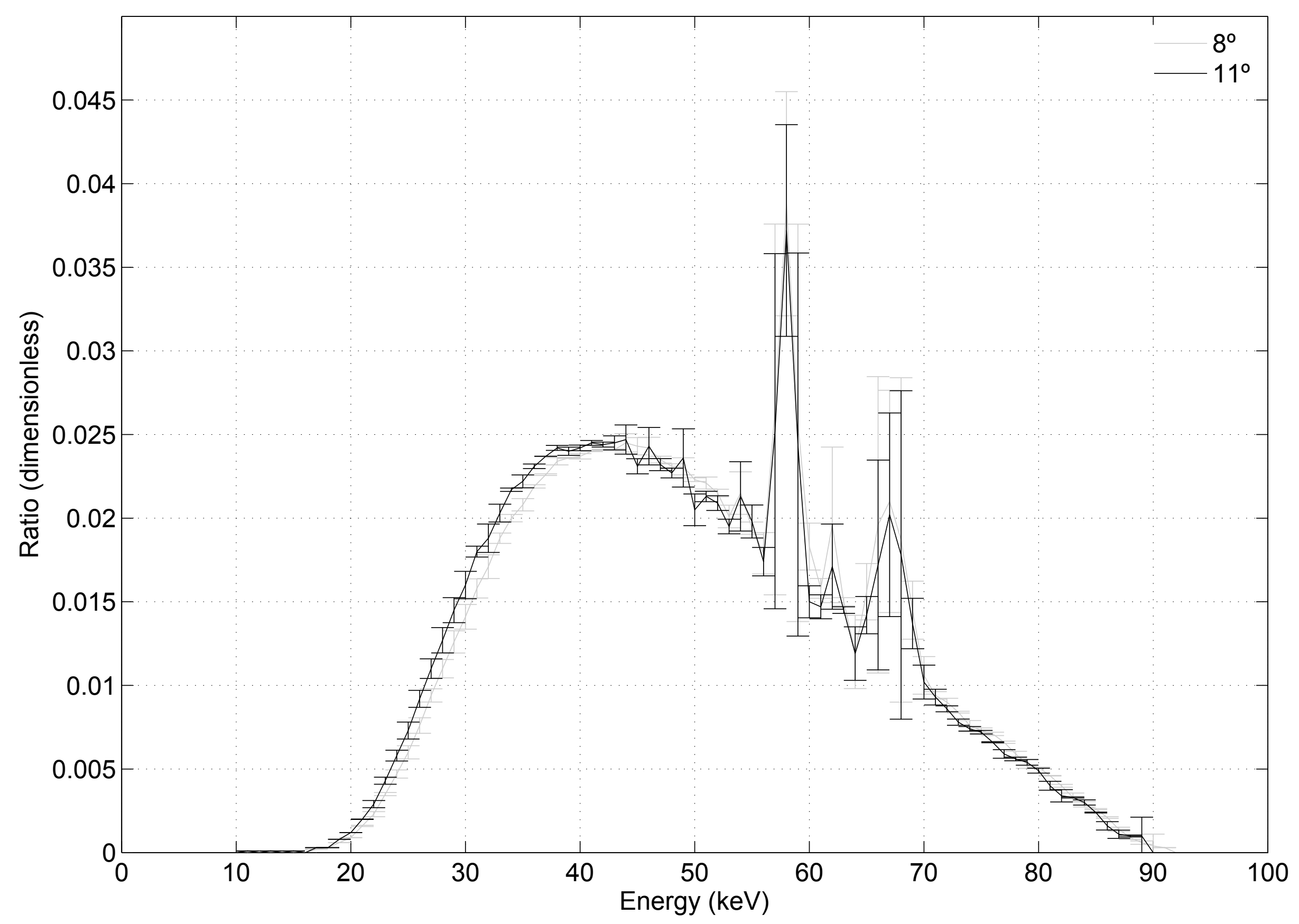




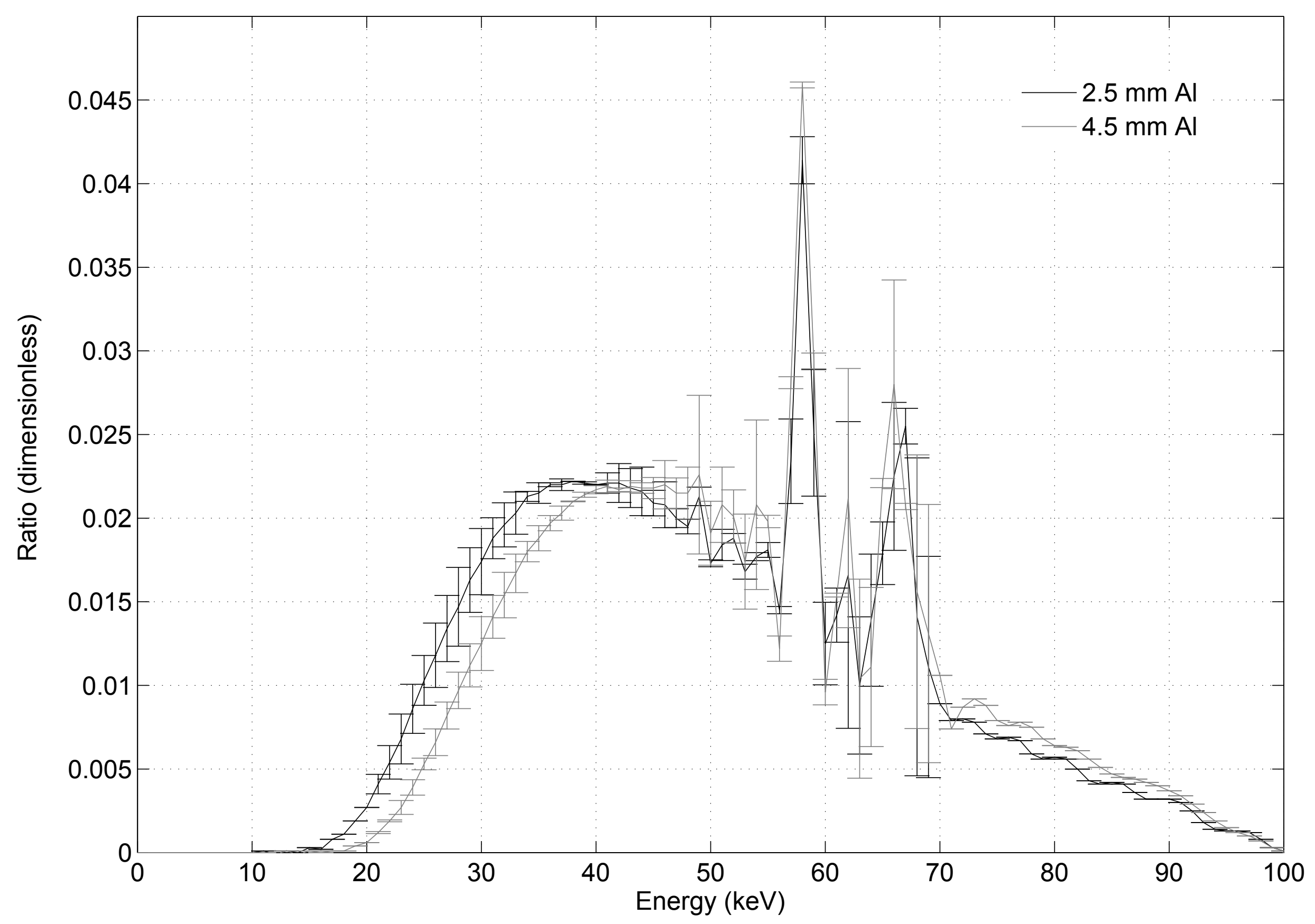

